\title{
JOSÉ ANGEL VALENTE Y LA BÚSQUEDA DEL CENTRO
}

\author{
Armando LOPEZ CASTRO \\ Universidad de León
}

En un breve y luminoso ensayo, escrito poco antes de morir, Lezama Lima sintetizaba así la trayectoria de Valente: "No creo que haya en la España de los últimos veinte años un poeta mús en el centro de su espacio germinativo que José Angel Valente, con la precisión de la ceniza, de la flor y del cuerpo que cac". Hay, en las palabras del "maestro cantor" la conciencia de una apuesta por lo absoluto. Tal es el sentido de toda búsqueda'.

Emprende, pues, la palabra esa búsqueda de lo imposible, intentando reconciliar al ser consigo mismo. Y aunque esta búsqueda se siga con cierta continuidad, se dan en ella detenciones significativas. Una de éstas tiene lugar en el poema "El autor en su treinta aniversario", que por sí solo comprende la segunda parte de La memoria y los signos (1966). En él hallamos estos versos:

Fl centro estii en lo gris

y en la inmovilidad, no en la acción.

El centro es al vacío.

Todo el poema constituye un retrato autobiográfico en el punto de vacío. El personaje que ha pasado por esa experiencia purgativa, a la que apuntan los primeros poemas del libro, ha encontrado una zona de vacio y se retrata en el gris, que es el color del centro, de la totalidad ${ }^{2}$. El gris mantiene una analogía con el punto cero, instante en donde convergen la nostalgia y la esperanza. Hay una renuncia de lo vivido y una apertura a la csperanza, una esperanza que va a traer algo que puede ser terrible ("Aguardo, / zarpa cruel de la esperanza, un día / tu bautismo sangriento").

Todos los poemas de la cuarta parte de La memoria y los signos presentan la destrucción en el amor como contro de la experiencia poética.

1 José Lezama Lima, "José Angel Valento: Un pota que camina su propia circunstancia", Revista de Occidente, $3^{n}$ ćpoca, Julio de 1976, núm. 9., pp. 60-61- La escritura de Lezama no ha sido tampoco ajena a la incorporación de un centro irradiante. Véase el prólogo de Valente, "El pulpo, la araña y la imagen", a Juego de las decapitaciones, Barcelona, Montesinos, 1982, pp. 7-13

2 Ei blanco y el negro convargen en el gris, que surge de la mezcla do ambos y funde los opuestos. El gris como color del contro aparece en algunas teorias actuales de la pintura, por ejemplo en Paul Klee, cuyo homenaje en el poema XVI de Treinta y siele fragmentos (1971) revela oste mismo sentimiento.

Para un análisis del gris como símbolo del centro véase cl estudio de Eva Valcárcel. El fulgor o la palabra encarnada. Imagenes y simbolos de la poesia última de Jose Angel Valente, Barcelona, PPU, 1989, pp. 73-81. 
El gris reaparece en "Esta imagen de ti".

Hablabas desde el centro del amor, armada de su luz, en una tarde gris de cualquier día.

Memoria de tu voz y de tu cuerpo mi juventud y mis palabras sean y esta imagen de ti me sobreviva.

Hay una interiorización del amor y una plegaria por su duración en la palabra. El personaje no está fuera del que habla, sino dentro y lo hace "desde el centro del amor". Lo que busca el poema es unificar la experiencia poética y la experiencia amorosa en un descenso radical hacia el centro de ésta.

Ligada está la destrucción a la inminencia en El inocente (1970). Do los muchos poemas que incorporan a su escritura la destrucción positiva que vertebra el conjunto, ninguno tan explícito como "Una oscura noticia", en el que se produce una asunción de la nada, de encuentro del hombre con lo divino.

Y tú, en medio,

tú solitario bajo las insignes galas

del otoño romano, vestido de amarillo,

taciturno y secreto,

aragonés o español de la extrapatria, ibas.

aniquilada el alma, a la estancia invisible.

al centro enjuto, Michele,

de tu nada.

Lo que más se reitera a lo largo del poema es la asociación del personaje con la idea de aniquilamiento. En el camino hacia la nada que lo es todo, el ser y la palabra se van desprendiendo de todas sus ligaduras hasta quedar libres de cualquier intención. Cuando la palabra alcanza ese estado de vacío, propiamente espirìtual, tiene el poder de su innombrable origen. Y es que la palabra poética lleva a una experiencia extrema, a ese punto quemado en que la experiencia de la escritura es una experiencia totalmente solitaria ${ }^{3}$.

Dentro de la serie de los Treinta y siete fragmentos (1971), unificada por el vacio positivo como símbolo del punto cero, hallamos este singular poema.

3 El poema "Una oscura noticia" supone el primer encuentro de Valente con Molinos. Después vino su edición de la Guía espiritual. Defensa de la contemplación, Barcelona, Barral Editores, 1974, cuyo proceso de elaboración él mismo ha explicado en "Breve historia de una edición", Variaciones sobre el pájaro y la red, Barcelona. Tusquets, 1991,pp. 258-278. Es también importante el artículo de María Zambrano, "Miguel de Molinos, reaparecido", Insula, núm. 338, pp. 3 y 4 


\section{XXXVI \\ (EL BLANCO)}

El arco armado y tenso une dos puntos del círculo a su centro.

El hemisferio del arquero en posición de tiro es la mitad visíble de la esfora completa que la lecha aún inmóvil ya ha engendrado.

Este fragmento remite a la anulación de la identidad y de la intencionalidad, a ese punto en que el arco en tensión hace que tirador, llecha y blanco sean la misma cosa. La imagen del arco en su tensión máxima, que vemos en el poema "Reaparición de lo beroico" y a la que Valente vualve en su introducción a la Guia espirilual de Molinos, sería ese punto extremo entre la vida y la muerte, capaz de encerrar el universo, cifra de la totalidad. De ahí que el movimiento de "la llecha aún inmóvil" ha de ser el del centro mismo, inmóvil y quicto. ¿Es la llecha la que da en el centro del blanco, 0 es el blanco quien acierta en la llecha?. Cuando se está en el centro, cuando se alcanza la plenitud en el punto de vacío, el arco y la llecha ya no son dos, sino uno. Ese estado de tensión, sin intención alguna, os el propiamente creadort.

El camino que lleva al centro es de difícil acceso. Desde las tradiciones indias hasta la céltica, se trata de una aventura espiritual, que consiste en sobrepasar lo visible para descubrir lo que bay detrás. lo único verdaderamente real. El relato "El señor del castillo", de El fin de la edad de plata (1973) es un rito del centro.

Ilabía una vez un rey que tenja tres hijas. La mayor era de oro; ]a del medio de óbano. A la menor nadie la conocia. El rey hizo (onstruir un onorme castillo en lo más escondido de los bosques que ocupaban el centro de su di]atado roino. El castillo no tenia ni puentes ni poternas ni atalayas ní catrada, y ori todo de cuarzos transparentes. Arrojaba en !a espesura silenciosa una insólita luz. Los hombres que llegaban hasta di se lacian transparentes y morían. El rey estaba triste y convocó a los sabios de su reina. Llamó primero al último, a aquel que vione siempre cuando todas las respuestas de los otros ya han sido vanas, ciegas o tardias. Y cuando íste enmudeció, el rey hizo decapitar a los restantes. Una vez que no hubo más sabios en al reino, ol rey antristecio de mís tristeza. Sus súbditos cubrieron de tierra negra los caminos perdidos. Se supo entonces que nadie que buscase aquel reino podría nunca llegar a sus confines. El rey llamó a su lecho de

- Para la técnica del tiro con arco como ejercicio espiritual, véase el estudio de Eugen Hlerrigel, Zen en el arte del tiro con arco, Buenos Aires, Kier, 6" ed., 1984. Ese estado de desprendimiento y no -intencionalidad, que supone el tiro con arco, of́rece una sorprendente afinidad con la escritura postica. 
muerte a sus tres hijas. La mayor era de oro; la del medio de ébano. A la menor gadie la conocía. Lloraron lágrimas de tierra y de ceniza. El llanto ritual detuvo el canto de las aves. El rey dividió el reino. A la mayor dio el aire, y a la del medïo, el agua; a la menor, el castillo transparente. Mas la hermana menor se negó a recibirlo. Tomó al roy su padre de la mano y bajaron así al contro luminoso de la tierra. Quedó el castillo arriba, en medjo de los bosques del dilatado reino, como luz o señal de lo menos visible, y cuantos hombres hasta él llegaban se hacian transparentes y morian.

El arquetípico castillo desempeña una función axial dentro del marco formulario de la narración. El viaje "al castillo transparente" revela una búsqueda espiritual, poética. En efecto, lo propio de la palabra poética es la exploración "de lo menos visible". ¿Cómo ascender si antes no se desciende?. Ese descenso "al centro luminoso de la tierra", que sería un descenso a la conciencia similar al "castillo interior" de Santa Teresa, convierte al castillo en simbolo de una realidad trascendental. Su transparencia es señal de trascendencia ${ }^{5}$.

En Interior con figuras (1976) empieza a darse una mayor radicalización de la materia entera, de la materia de la experiencia y de la materia verbal. Se trata de que sea la propia materia la que hable, la que de manifestación de su propia posibilidad. Esta interiorización de la materia lleva al poeta a concentrarse, a encontrar su propio centro. También el árbol va ligado al simbolismo del centro y es lugar de paso entre lo visiblo y lo invisible.

\section{ELEGIA, EL ARBOL}

El ârbol pertenecía por la copa a lo sutil, al aire y a los pajjaros. Por e] tronco a la germinación y a todo lo que une lo celeste con los dioses del fondo. Por la raiz oscura, a las secretas aguas. La copa dibujaba un amplio semicírculo partido. Porque también el ărbol era portador del fuego, herido por el rayo, el árbol. Como otras cosas mayores que nosotros, estaba el árbol no en la ciudad, sino en el mundo, más cierto que ella misma, que aún la circundaba. Arbol. Ciudad. El árbol en lo alto, sobre la lentitud de la subida. Nos llevaban a él, pensábamos, su propia suliciente soledad o su

sobre esta exploración poética "de lo menos visible", véase el estudio de Dionisio Cañas, Poestá y percepción (Francisco Brines, Claudio Rodriguez y Josét Angel Valente), Madrid, Hiperión, 1984, pp. 182-184

Para la convergencia de la experiencia poética y mística en lo espacial, a propüsito de este relato, debe tenerse en cuenta el ensayo de Amparo Amorós Moltó “El espacio en la poesía de José Angel Valente", Insula, núm. 412, pp. 1 y 10

El castillo como espacio sagrado, como metáfora del viaje al centro, está presente en toda la tradición occidental. Con respecto a esto, téngase en cuenta el estudio de Malph Metzner, Las grandes metáforas de la tradición sagrada. Barcelona, Kajrós, 
belleza. Soledad o belleza, santidad, forma que en la cercaníl del dios reviste lo viviente. El árbol, nos dijeron, fue talado. El árbol, no de la ciudad, sino del mundo, más real, que entonces aún la circundaba. Quien visite el lugar acaso sepa que aquel árbol no podía morir, que en el lugar del árbol para siempre hay, igual al árbol, en la posición antigua del orante, un hombre, igual al árbol, con los pies en la tierra, pero menos visible, la cabeza y los brazos, con las manos abiertas, alzadas hacia el cielo, copa, tronco, raiz, para que desde lo oscuro suba lo oscuro al verde, al rojo, y a su vez el fuego regrese de lo alto a la matriz, al centro imperdurables.

La función principal del arte es la de transformarnos. El poeta se reconoce en ese árbol "herido por el rayo", que alude al famoso roble situado a las afueras de Orense, porque "no estaba en la ciudad, sino en el mundo", árbol del centro del mundo o imagen cósmica, y porque el hombre, "igual al árbol", debe convertirse en algo duradero. En las religiones arcaicas, hay una unión primordial entre el árbol y el hombre. El árbol es símbolo del universo y el hombre asociado al árbol participa de la regeneración cósmica. Por eso, dentro del asentimiento al eterno ciclo de la naturaleza en que el poema consiste, el poeta tiene la misma posición lisica del orante antiguo, que es la posición del hombre de pie, y se hace mediador en la situación del tronco. Esta función mediadora es la que mejor define a la palabra poéticat.

En Material memoria (1979) la palabra desciende hacia la interioridad de la experiencia hasta hacerse memoria de la materia. La equivalencia entre la memoria de la materia y la memoria de la palabra revela que la materia es para el pocta el origen de toda posible forma. Esta materia en formación, este movimiento de lo informe hacia la forma, engendra un vacio, que es inminencia de significación. De ese vacío o espera fecunda surge la palabra

\section{PALABRA}

Pulabra

hecha de nada.

Rama

en el aire vacío.

"Ese árbol perdura en la memoria, se hace inmortal, porque es antes que nada eje a centro del mundo. Señala Mircea Eliade: "La variante más extendida del simbolismo del Centro es el Arbol Cósmico, que se halla en medio del Universo, y que sostiene como un eje los tros Mundos", en Imágenes y simbolos, Madrid. Taurus, 2* ed., 1979, p. 47

Paul Klee, en su Diario, ha comparado al artista con la situación del tronco. Para el complejo simbolismo del árbol en las religiones arcaicas, donde es el universo y participa de él, véase el estudio de Charles Hirsch, El árbol, Barcelona, Plaza y Janés, 1989. 
ARM NDDO LOPEZ CASTRO

5 Ala

sin pájaro.

Vuelo

sin ala.

Orbita

10 de qué centro desnudo

de toda imagen.

L.4Z.,

dondẹ aún no forma

su innumerable rostro lo visible.

La palabra abre aquí un vacio. Y en el vacio, la necesidad de una forma. Es acaso la nada el centro donde se cumple la unión de la materia con la Corma. De ahí que la palabra, para decir la materja, tenga que despojarse de sus formas. La prolongada aposición en forma negativa es, junto al desplazamiento de las dos últimas estrofas, rasgo distintivo del poema. La palabra asume la experiencia de la nada o del vacio sin poder desprenderse de ella. Y la palabra, sostenida por el silencio, queda disponible. "Pulabra / hecha de nada" o de silencio, pues el silencio es tal vez la única posibiljdad de que la palabra pueda cumplirse como tal, según sabe el poeta después de haber leído a Molinos ${ }^{7}$.

La reflexión de Tàpies sobre la materia no es ajena a la visión poética de Valente. Por eso, los "Cinco fragmentos para Antoni Tàpies" no pueden separarse del resto. Tal vezuno de los símbolos que mejor exprese la totalidad del cosmos sea el simbolo de la cruz, "arbol axial, eje de la extensión y de la altura, la cruz es el símbolo unificador de la materia viva del mundo", como nos dice el poeta en el IV de su fragmentos. La cruz, igual que el airbol cósmico, significa la materia del mundo en su totalidad".

7 Este poema no va dedicado a María Zambrano de forma casual. Ella misma, en su artículo "Calvert Casey, el indefenso entre al ser y la vida" (Quimera, nóm. 26, diciembre de 1982 , pp. 56-60), nos recuerda la frase del escritor cubano, ${ }^{4}$ Maria, qué Himno más Vódico a la Nada!", despues de su lectura de la Guía de Molinos. También Valente, en su articulo ya citado "Brove historia de una adición", recuorda que el origen de la Guía se debe a su amistad con Casey. La común lectura de Molinos se conjuga con la cita de Lezama que abre el libro, donde el aire o la luz son "el primer animal visible de lo invisible”, y con la tradición mistica. particulatmente San Juan de la Cruz, donde la visión de la nada o del vacío ocupa un lugar central.

Por otra parte, los ensayos de Andrés Sünchez Robayna. "Arte de la composición del silencio" (El Pais, 18 de marzo de 1979, p. VII) y de María Payeras Grau, "El don de lo imposible" (Revista Espacios. Invierno de 1991, pp. 34-38), insisten en esta desposesión radical de la palabra.

a Aunque la iconografía cristiana se ordena en torno al símbolo de la cruz. 
El sentido cósmico de la cruz está presente en Tres lecciones de tinieblas (1981), poema único formado por dos ejes: el horizontal de la historia y el vertical de las letras. La destrucción del discurso histórico es la que hace posible el regreso a la palabra inicial y absoluta. Lo discontinuo debe convertirse en continuo. La penetración en la materia, la exploración de la matriz, equivale a lograr la integración, a situarse en ese centro no manifestado que contiene todas las manifestaciones posibles. Ese punto esencial es el que señala la novena letra.

\section{TET}

La sangre se hace centro y lo disperso convergencia: todo es reabsorbido desde la piedra al ala hasta el lugar de la ganeración: las aves vuelan en redondo para indicar el centro de lo cóncavo: el mundo se retrae a ti: porque el vientre ha de ser igual al mundo: engéndrame de nuevo: hazme morir de un nuevo nacimiento: respírame y expülsame: animal de tus aguas: pez y paloma y sierpe.

Todo tiende aquí a precipitarse o a ser reabsorbido en el centro de esa materia generadora. Hacia el final del SÉFER YETZIRÁ o LIBRO DE LA FORMACIÓN, a propósito de Abraham y citando a Jeremías, se dice" "Antes que te formase en el vientre te conocí, y antes que nacieses te santiliqué". El vientre, el seno materno, es el lugar de la formación del hombre. Una vez establecido el reingreso en el centro, "La sangre se hace centro y lo disperso convergencia", se entiende la identilicación central, "porque el vientre ha de ser igual al mundo", y a partir de ella, el nacimiento a la vida cósmica, "respíramo y expúlsame". La mujer o su matriz engendra al hombre como la materia infinita del mundo genera una escritura abierta, cuyos signos lingüisticos más visibles son la novena letra, que señala un punto central entre lo alto y lo bajo, cuya reiteración es manifiesta a lo largo del poema ("centro", "convergencia", "lugar de la generación", "centro de lo cóncavo"): el "tu" dialógico entre el poeta y la inlinita materia del mundo; el signo grálico (:), que revela una continuidad on la escritura de esa materia viviente; las continuas formas exhortativas ("engéndrame", "respírame", "expúlsame", "hazme morir"). que indican el poder de esa materia fecundante; $y$. por último, los tres símbolos que cierran el poema ("pez y paloma y sierpe"), esto es, las aguas, el aire y la tierra, presentes en todas las tradiciones. Lenguaje sagrado, lleno de símbolos, testimonio de que el hombre ha gozado alguna vez de la unidad central original, lugar propio del

conviene recordar que la cruz, no se utiliza como símbolo de Cristo hasta después del siglo XIV. Al señalar los cuatro puntos cardinales, la cruz signifíca la totalidad del cosmos, es un símbolo universal. Véase el estudio de R. Guénon, Le symbolisme de la Croix, Paris, 1931. Hay traducción ospañola, Barcelona, Ediciones Obelisco, 1987 
hombre que la palabra se esfuerza por reencontrar y descilrar".

La constante implicación entre la materia, el amor y la palabra, que se advicrte en Material memoria, Estancias y Tres lecciones de tinieblas, no hace más que agudizarse on los libros siguientes.

Mandorla (1982) es también otra figura geométrica que representa el símbolo del centro. La mandorla concentra: protege de lo exterior, de la dispersión. Tiende, pues, la palabra a concentrarse, a hallar su propio centro, a instalarse en lo real. Esta ausencia de lo real es lo que revela el mito del Graal, ligado al simbolismo del Centro.

\section{GRAL.}

Respiración oscura de la vulva.

En su latir latia el pez del légamo y yo latía en ti.

Me respiraste

5 en tu vacio lienoy yo latía on ti y en mi latían

la vulva, el vorbo, el vértigo y el centro.

Lu concavidad del Graal representa para ol poeta la inmanencia de la materia on que eros y poesia coinciden. Porque el movimiento hacia el centro de la materia-espíritu. donde la palabra y el cuerpo del amor son lo mismo, es también un movimiento hacia el centro de la interioridad. La matriz de la mujer se equipara con la matriz del universo. La estancia en la mitriz como centro de la vida, del cosmos y de la creación, es un estado central, intemporal, en el que tienen lugar todos los nacimientos. La mujer se identifica con la Naturaleza. La ley profunda de lo femonino es el ritmo natural, que puede reunir en si el movimiento y la identidad. La Mujer empieza dondo acaba la Historia. Asociada al ritmo circular de la Naturaleza y no al lineal de la Historia, la Mujer constituye un estado absoluto de la materia. Siendo la Mujer la Naturaleza, es necesario que el hombre se remita a ella. Todo ese dominio de lo femenino conlleva la reabsorción y la manilestación, que son los dos movimientos de la respiración. El "vacio lleno" de la vulva es, metafóricamente, la plenitud de la materia. Ser

'El movimiento de la palabra hacia la materia infinita del mundo, que comienza ya en los poemas de Interior con figuras, es el de un desprenderse cuanto es posible para ser una con la materia $e$ ir apareciendo con ella. Una comunión se produce de inmediato entre esa materia madre y la palabra sostenida por ella. $Y$ asi llegamos a sentir en estos poemas que es el Cosmos el que respira, vive y se mueve. Vétse el urtículo de María del Carmen González Marín. "Una lectura del cosmos; el último libro de José Angel Valente" , Insula, núm. 413, p. 7. También es importante el anälisis que de este texto hace Milagros Polo en su estudio José Angel Valente: poesia y poemas, Madrid, Narcoa, 1983, pp. 217-221 
respirado por la matriz es conjurar toda ruptura de la materia, quedar inscrito en esa centralidad on la que ya no hay diferencia entre la materia y la voz del poema, como no la hay entre "la vulva, el verbo, el vértigo y ol centro". La fórmula diseminativo-recolectiva del último verso, además del desplaza-miento que subraya el protagonismo de la matriz ("Me respiraste (en tu vacío lleno"), no sólo es signo de sabia construcción, sino también expresión de una escritura matriz, de un lenguaje que es suspiro o aliento. Sin duda, la Mujer se convierte aquí en objeto religioso. Es como si la concavidad del Graal, que pone en escena lo absoluto, representase el vacio místico lleno de posibilidades ${ }^{10}$.

La reabsorción en la matriz implica un regreso a la indistinción primordial, a la indiferenciación. Y así, lo primero que esta unidad de origen reclama es una escritura sin límites, una abolición de los géneros. Esta ausencia de géneros, que podría trasladarse a su obra total en virtud de la mirada originaria que la habita, resulta evidente en Elfin de la edad de plata (1973) y en Nueve enunciaciones (1982), que pertenecen al territorio de la creación, y en Las palabras de la tribu (1971) y La piedra y el centro (1982), en los que se da un intento de llevar al ensayo la tensión de la poesía. En el primer ensayo de este último libro y que sirve de título al conjunto aparece la unidad simbólica de la piedra y el centro, "La piedra y el centro son, on realidad, lo mismo", que tiene sólidos apoyos en todas las tradiciones. Al final de "Objetos de la noche", primer poema de Material memoria, el poeta expresa su deseo de una escritura total: "tender la red al fondo / vislumbrar en lo oscuro / el poema o la piedra, / el don de lo imposible". Con esa identificación, "el poema o la piedra", el poeta intenta que la escritura poética sea representación del centro, de la totalidad.

Entre las representaciones simbólicas de la piedra como centro cósmico y punto de unión con lo divino, destacan la piedra que sirve de cabecera a Jacob, la piedra nogra de la Meca, el omphalos de Delfos y el quicunce de la mitología azteca. Lo divino se manifiesta por medio de la pied ra y la piedra es un signo de lo divino".

10 El hallazgo de esa materia encarnada, del amor y de la palabra, sería también un conocimiento de lo real . Como señala Miguel Mas a propósito de este poema, "El elemento femenino opera entonces como palabra auténtica reencarnada, unidad de maleria y espiritu al estilo da la Mística". La escrilura material de José Angel Valente, Madrid. Hliperión, 1986, p. 63 En este sentido, el estado de transparencia de la materia interiorizada vendria dado por la estancia en el centro, ya presente en el mito dol Graal: "La aparición del Granl en el centro do la Mesa Redonda (con doco caballeros), símbolo de la totalidad, lo caracteriza como simbolo del centro", nos recuerda Joan Ramón Resina en su estudio La bísqueda del Grial, Barcelona, Anthropos, 1988, pp. 339

"Sobre el caraicter sagrado de las piedras, pueden consultarse los estudios de Mircea Eliade, "Las piedras sagradas: epilanías, signos y formas", Tratado de historia de las religiones, I, Madrid, Cdiciones Cristiandad, $6^{*}$ ed. 1974, pp. 253-278; y de Gutierrez Tibon, "El ombligo de piedra", El ombligo como centro cósmico. Una contribución a la historia de las religiones, Méxjco. Fondo de Culura Económica, 1981, pp. 261-303 
La imagen arquetípica de la piedra central, que establece el equilibrio del mundo, lija y concentra la unidad del origen, esa unidad primero tenida, perdida luego $y$ que ha de hallarse nuevamente. $Y$ es justamente en esa vuelta al origen donde la piedra y el poema coinciden. Porque lo que hace la copla anónima es reconducir la separación a la unidad: "Fui la piedra y fui el centro / y me arrojaron al mar / y al cabo de largo tiempo / mi centro vine a encontrar". En rigor, la piedra y el poema son una experiencia de la unidad. La piedra es una materialización del centro y el arquetipo centropiedra es una manifestación de la memoria colectiva. Ser es recordar y recordar es liberarse del tiempo para instalarse en el origen, en lo real. Esa memoria colectiva lo que hace es darnos una continuidad en la experiencia, una identidad. La piedra del centro es la piedra de la fundación, en la cual está grabado ol Nombre divino según una conocida leyenda hebrea. La palabra remitiria, pues, al Nombre del que nace todo lenguaje. El poema, como la piedra, sería una representación de la unidad de los nombres, del punto cero que contiene la totalidad del lenguaje. El sentido general de este ensayo es que, para la visión de la escritura como experiencia de la totalidad, es de capital importancia la preservación del centro.

Lo singular del centro es la unilicación de la experiencia. Se trata de saber vivir con él, de decir con él una sola materia: la palabra y el amor en Mandorla (1982), cl diálogo con el cuerpo del amor en El fulgor (1984), la percepción de la materia infinita del mundo en $A l$ dios del lugar (1989). Sin la convergencia de espiritu y materia en un punto central único, identidad que tan desarrollada tienen los orientales, dilícilmente podríamos leer estos poemas. Porque cada uno de ellos nos abre hacia una interioridad que lo trasciende todo y que hay que aceptar. Estado de espera, propio de lo poético. No a otra cosa se habria reforido el poeta en Material memoria: "Crear lleva el signo de la feminidad. No es acto de penetración en la materia, sino pasión de ser penetrado por ella". La palabra penetra el cuerpo del amor y se impone al poela, que no hace más que seguir su huella en este poema de El fulgor.

\section{VIII}

Vuelvo a seguir ahora

tu glorioso descenso

hacia los centros

del universo cuerpo giratorio,

una vez más ahora,

desde tus propios ojos,

tu larga marcha oscura en la materia

más fulgurante del amor.

La noche. 
al absoluto vértigo, la nada.

El instante pone a prueba la realidad. Lo absoluto del instante ("ahora") nos hace ver que la experiencia erótica, poética y mística constituyen una sola experiencia. Porque ese proceso de descondicionamiento, en que la palabra se vacia de toda forma para llenarse con la plenitud del amor, es constitutivo de toda experiencia mistica. No hay palabra que no experimente el viaje del alma a través de "la noche oscura", el descenso a las sombras y la posterior salida a la luz. El deseo de poseer es la negación del ser. Por eso, la cita es aquí con la noche, centro del poema, y el gozo de la unión es un espacio lleno de vacio. El amor empuja a la palabra para que lo destruya $y$ él pueda renacer en el instante del poema. Descondicionar las palabras es devolverlas su posibilidad.

En Al dios del lugar (1989) hay una aproximación a la materia del mundo. que sería un modo de acercamiento a la Palabra perdida, origen de toda poesía y anterior a la historia. Fuente de todo lenguaje, la palabra del dios es palabra absoluta que sólo se da en el sacrificio del poema, lugar de la relación materia-espíritu, reproducción del ciclo cósmico. Por eso, el poeta recurre aquí a un lenguaje especílicamente religioso, que está més allá de las palabras y en el que se ha operado una abolición delsentido. La conjunción pájaro-palabra se verifica como un mutuo comparecimiento en ese estado de suspensión propio de la escritura poética.

Suspendido del canto, en el centro o en el eje celeste de la tarde. el pájaro.

El vuelo del pájaro, como el del poeta, es siempre solitario. Captada en su centro ("en el centro o en el eje/celeste de la tarde", "El color era el gris", "En el vacío/y la inmovilidad"), la imagen del pájaro es una imagen cósmica. Pájaro absoluto, palabra absoluta. Lo que intenta el poeta es crear una palabra que sea igual a ese pajjaro de las antiguas narraciones, que nos hace olvidar el tiempo y el espacio para proyectarnos al viaje ritual del universo. El paijaro da a la palabra la plena medida de su cosmicidad. El pájaro sería, pues, la cifra del canto y del mundo. Comprender el lenguaje de los pájaros es aprender el lenguaje del silencio, de la poesia ${ }^{12}$.

12 La imagen central del pájaro, que aparece en el salmo 102, en San Juan de la Cruz, en Leopardi, en lilke, formaria parte, según Valente, "de una tradición poética en la que Oriente y Occidente han de volver a encontrarse", en Variaciones sobre el pajaro y la red. Opus Cit.. p. 162. Valente ha captado el pajjaro como una imagen de lo absoluto en analogía con la pajabra poética. En este sontido, son importantes sus ensayos "Las condiciones del pájaro solitario", "Dove vola il camelonte" y "Sobre la lengua de los pájaros", todos ellos dentro del libro citado. 
El crecimiento hacia el centro, lugar donde alma y cuerpo convergen y se unifican, es lo que constituye la estética del poema. En tal estado de formación, la palabra todavía no ha sido dicha, No amanece el cantor (1992). Libro denso y complejo, que atrae, en una primera aproximación, por la extremada tensión de su lenguaje. En ese lento proceso de concentración de la escritura, en el que todo se disuelve como absorbido en la muerte, hallamos este poema nuclear.

El centro es un lugar desierto. El centro es un espejo donde busco mi rostro sin poder encontrarlo. ¿Para eso has venido hasta aqui?? ¿Con quién era la cita? El centro es como un círculo, como un tiovivo de pintados caballos. Entre las crines verdes y amarillas, el viento hace volar tu infancia. -Deténla, dices. Nadie puede escucharte. Músicas y banderas. El centro se ha borrado. Estaba aquí. en donde tú estuviste. Veloz el dardo hace blanco en su centro. Queda la vibración. ¿La sientes todavía?

El lenguaje del poeta, su manera de expresarse, nos ofrece una iluminación de su propia experiencia. La progresiva disolución que aquí se observa ("El centro es un lugar desierto", "El centro es un espejo donde busco mi rostro sin poder encontrarlo". "El centro se ha borrado") tiene por objeto liberarse de todas las ligaduras, de modo que la palabra, sumergída en sí misma, quede en un estado de tensión, sin intención alguna, y se halle en la posibilidad de su origen. Palabra, pues, del desierto, del no lugar, según nos dice Valente hablando de Jabès. Al final, cuando todo se ha borrado ("El viento hace volar tu infancia”), el arco, la flecha y el blanco están unidos y ya no es posible separar nada ("Veloz el dardo hace blanco en su centro"). Lo único que se siente es la pura vibración ("Queda la vibración"), el despliegue del lenguaje como brotando de la nada. De ese centro oscuro o estado de vacío, en el que el poeta y el hijo muerto ya no son dos sino uno, surge la palabra poética. A ese imposible diálogo con al hijo correspondería el indecible decir en que lo poético se funda. Y es que el vacío es siempre espera de la palabra. El Centro, que es ante todo el Principio, lo Real absoluto, la Unidad primera que todo lo contiene, ne está en ninguna parte, es lo no manifestado y que, debido a su no manifestación, contiene todas las manifestaciones posibles. La experiencia unificadora del centro, que se encuentra en todas las religiones orientales, es el punto de quietud y de la receptividad, el punto desde el que es posible ver la totalidad de las cosas. Valente ha confesado repetidas veces que siente la fascinación del centro invisible pero constituyente, cuyo descubrimiento es indispensable para percibir la realidad oculta, y que esa fascinación del centro inaprensible tiene que ver mucho con la escritura entendida como totalidad. En ese centro unilicador, punto de contacto del cielo y de la tierra, tiene lugar el retorno a lo primordial, 
a la palabra liberada del lenguajo ${ }^{13}$.

El camino al centro es también el regreso al origen, en el que siempre se estuvo, y todos los grandes mitos aluden a la dificultad de alcanzarlo. Ulises, Parsifal, todos los que buscaron el Graal, hicieron un largo camino para encontrar un lugar que nunca se ha abandonado. Su experiencia nos dice que debemos reconquistar el centro a través de un arduo ejercicio en el arte de olvidarnos de nosotros mismos. ¿Acaso no es la escritura esta sostenida pobreza?.

Unificar el pensamiento y la palabra es tarea de muchos años o tal vez de toda la vida. Los años de aprendizaje desembocan, como es natural, en la incorporación de los distintos fragmentos a un centro irradiante, cuya claridad de razón de todos ellos. Tal unidad parece ser aquí la de la experiencia religiosa, la del Verbo que ha concentrado en sí todas las palabras. En su sentido más profundo, religión y poesía se debaten entre la pérdida y la recuperación, buscan reunir lo disperso, hallar la Palabra perdida.

Perder el centro es perder la unidad del origen. Buscarlo es recuperar lo absoluto, lo real. A medida que la unidad de la obra se despliega, los diferentes géneros que la integran, el poema, el ensayo, la narración, son como radios de un centro al que terminan por incorporarse y en el que ya no se distinguen. El centro sería, pues, el lugar de la unidad, de la escritura. Estar en el centro es tener una experiencia de lo absoluto y la poesía debe ser una apuesta por lo absoluto.

${ }^{13}$ Más que una posición geométrica, al modo de Pascal, el centro es un lugar sagrado, taj como advierten M. Eliade y R. Guénon. El camino hacia cl centro, hacia esta Unidad primera buscada por la poesia, es un camino de aprendizaje, una configuración de distintos descubrimientos que se integran. Porque los distintos radios nacen de un centro y convergen en un centro irradiante: "La vir tud del centro es atraer. recoger en torno todo lo que anda disperso. Lo que va unido a quo el centro sea siempre inmóvi]", dice Maria Zambrano en "El centro y el punto privilegiado", recogido en Claros del bosque, Barcelona, Seix Barral, 1977. Y el lenguaje ha de ir a dar a ese centro inmóvil y quieto, al silencio del punto cero, de donde sólo nace el canto. 